\title{
Nomogram predicting survival to assist decision-making of radical prostatectomy in patients with metastatic prostate cancer
}

\author{
Kan Wu ${ }^{1 \#}$, Yongquan Tang ${ }^{2 \#}$, Yanxiang Shao ${ }^{1}, \mathrm{Xiang}^{{ }^{1}}$ \\ ${ }^{1}$ Department of Urology, Institute of Urology, West China Hospital, Sichuan University, Chengdu, China; ${ }^{2}$ Department of Pediatric Surgery, West \\ China Hospital, Sichuan University, Chengdu, China \\ Contributions: (I) Conception and design: X Li; (II) Administrative support: X Li; (III) Provision of study materials or patients: K Wu, Y Tang; (IV) \\ Collection and assembly of data: K Wu, Y Tang, Y Shao; (V) Data analysis and interpretation: K Wu, Y Tang, Y Shao; (VI) Manuscript writing: All \\ authors; (VII) Final approval of manuscript: All authors. \\ \#These authors contributed equally to this work. \\ Correspondence to: Xiang Li, MD, PhD. Department of Urology, Institute of Urology, West China Hospital, Sichuan University, 37 Guoxue Lane, \\ Chengdu 610041, China. Email: xiangli.87@163.com.
}

Background: Radical prostatectomy (RP) has heterogeneous effects on survival of patients with metastatic prostate cancer (mPCa). A reliable model to predict risk of cancer-specific mortality (CSM) and the potential benefit derived from RP is needed.

Methods: Patients diagnosed with mPCa were identified using the Surveillance, Epidemiology, and End Results database (2004-2015) and categorized in RP versus nonlocal treatment (NLT). Based on the Fine and Gray competing risks model in 8,463 NLT patients, a nomogram was created to predict CSM in mPCa patients. Decision tree analysis was then utilized for patient stratification. The effect of RP was evaluated among 3 different subgroups.

Results: A total of 8,863 patients were identified for analysis. Four hundred (4.5\%) patients received RP. The 5-year cumulative incidence of CSM was $52.4 \%$ for the entire patients. Based on nomogram scores, patients were sorted into three risk groups using decision tree analysis. In the low- and intermediate-risk group, RP was found to be significantly correlated with a $21.7 \%$ risk reduction of 5 -year CSM, and $25.0 \%$ risk reduction of 5-year CSM, respectively, whereas RP was not associated with CSM in high-risk group (hazard ratio $=0.748,95 \%$ confidence interval 0.485-1.150; $\mathrm{P}=0.190$ ).

Conclusions: We developed a novel nomogram and corresponding patient stratification predicting CSM in mPCa patients. A newly identified patient subgroup with low-, and intermediate-risk of CSM might benefit more from RP. These results should be further validated and improved by ongoing prospective trials.

Keywords: Metastatic prostate cancer (mPCa); radical prostatectomy (RP); nomogram; cancer-specific mortality $(\mathrm{CSM})$

Submitted Aug 12, 2020. Accepted for publication Dec 15, 2020.

doi: $10.21037 /$ tau-20-1166

View this article at: http://dx.doi.org/10.21037/tau-20-1166

\section{Introduction}

Metastatic prostate cancer ( $\mathrm{PCa}$ ) has a poor prognosis, and the incidence of metastatic PCa has increased significantly in the United States (1). Currently, the main treatment modality for newly diagnosed metastatic PCa is androgen deprivation therapy (ADT), followed by chemotherapeutic agents (2). Unfortunately, these treatment options are quite limited and have a heavy economic burden (3). For several oncologic entities, maximal cytoreductive surgery has shown a significant improvement in the survival of patients with metastatic disease (4-6). Therefore, radical prostatectomy (RP) is considered a potential option for prolonging survival in patients with metastatic PCa. 
Data from a series of population-based studies demonstrated that definitive local treatment, either surgical intervention or radiation therapy, was associated with improved survival in metastatic PCa patients, even in the presence of metastases (7-10). Additionally, previous studies and a recently published phase 1 study on cytoreductive surgery have confirmed that RP appears to be safe and has no extra complications in select patients with metastatic PCa (11-13). Obviously, some patients with distant metastases may benefit from RP, but there is limited data about which subgroups may benefit from aggressive surgery.

Therefore, producing a clinically applicable model to identify the selection criteria and optimal candidates for RP is the center of the issue. The nomogram is a convenient tool that can quantify individualized risk prediction and has value in risk stratification. Some researchers have proved that nomograms can provide precise risk prediction in multiple cancer entities $(14,15)$. Despite several prognostic nomograms have been constructed for predicting survival of PCa patients (16), the nomogram guiding the selection of metastatic PCa patients for RP is still lacking.

Thus, we utilized a population-based database to develop a prognostic model for the stratification of patients and to further identify a patient subset who may be more likely to benefit from RP in patients with metastatic PCa. We present the following article in accordance with the TRIPOD reporting checklist (available at http://dx.doi. org/10.21037/tau-20-1166).

\section{Methods}

\section{Study population}

Data were derived from the National Cancer Institute's Surveillance, Epidemiology, and End Results (SEER) database which collects and publishes cancer-specific outcomes for approximately $28 \%$ of the American population. Using the SEER database, we identified patients diagnosed with metastatic PCa between 2004 and 2015. The SEER data were open available and patients' records are anonymous, therefore, this study was deemed exempt from review by our institutional review board. The study was conducted in accordance with the Declaration of Helsinki (as revised in 2013).

Inclusion criteria included the following: (I) patients diagnosed with prostate adenocarcinoma (primary site code: C61.9, and histology code: 8140); (II) patients diagnosed with radiographic, or pathological confirmation of metastasis; (III) patients were between 35 and 90 years at initial diagnosis, and follow-up $>3$ months. Patients with missing information on metastatic substages (M1a, M1b, or M1c), T stage, $\mathrm{N}$ stage Gleason score, prostate-specific antigen (PSA) value, or survival status were excluded in the cohort. Likewise, cases treated with other types of surgery (e.g., cryotherapy, or transurethral resection) or radiation therapy [e.g., external beam radiation therapy (EBRT), or brachytherapy], as well as those with unknown radiation therapy or surgery information also were excluded.

Patients were divided into two groups based on treatment type: RP group (surgery site codes 50 or 70) vs. nonlocal treatment (NLT) group (defined as ADT or observation).

\section{Demographic and clinical variables}

Study variables included age, race, marital status, PSA, Gleason score, tumor stage, treatment modality (surgery, or radiotherapy), survival months, vital status and cause of death (based on the SEER cause-of-death classification). Clinical variables and survival outcomes of patients were collected by two independent authors (K Wu and Y Tang).

\section{Statistical analysis}

Differences in clinicopathologic characteristics according to RP and NLT were examined using Chi-square test for categorical variables. Multivariable Fine and Gray competing risks regression analyses for identifying variables associated with cancer-specific mortality (CSM) was performed and expressed as hazard ratio (HR) with $95 \%$ confidence interval (CI). Non-prostate cancer related death was treated as competing risk event for $\mathrm{PCa}$-specific death.

The nomogram predicting CSM was built in patients treated without RP. All variables in the multivariable competing risks regression analyses were used to build a nomogram model for predicting CSM (age, PSA, Gleason score, $\mathrm{T}$ stage, $\mathrm{N}$ stage, and $\mathrm{M}$ stage). To account for PSA distribution differences, logarithmic PSA transformation was used. Internal validation of the nomogram was performed using the Harrell's concordance index (C index) and area under the time-dependent receiver operating characteristic curve (AUC).

According to the nomogram scores, decision tree analysis (Chi-Square automatic interaction detector) was utilized to identify the optimal cutoff points for dividing patients into three prognostic groups (i.e., the low-, intermediate-, and high-risk groups). 
Table 1 Baseline demographic and clinical characteristics of the patients

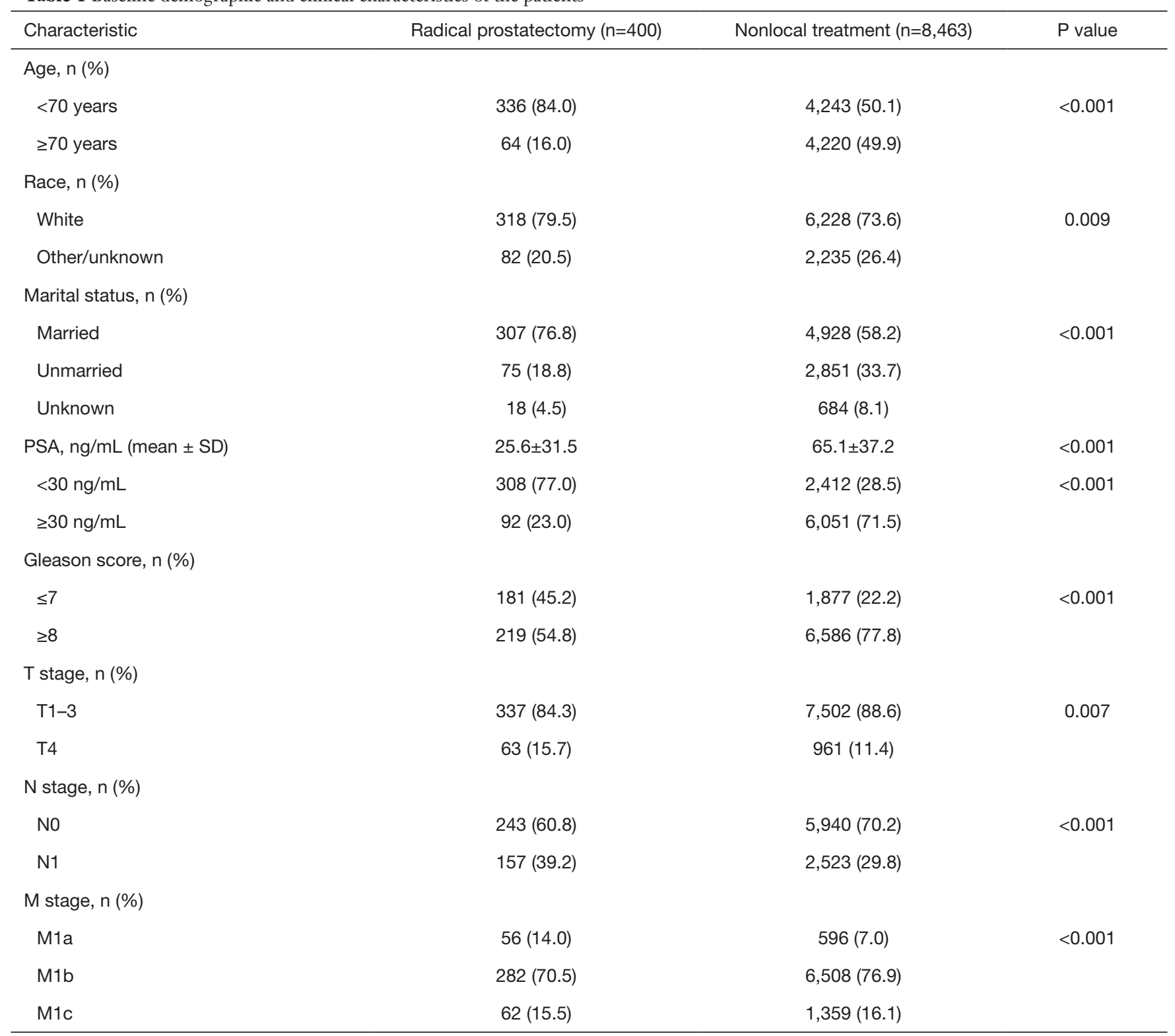

PSA, prostate-specific antigen; SD, standard deviation.

P values $<0.05$ were determined as statistically significant. All statistical analyses were performed with SPSS software version 23.0 (IBM, Armonk, NY, USA), and R software (version 3.5.1; R Foundation).

\section{Results}

A total of 8,863 eligible patients with metastatic PCa were enrolled in the study cohort. Among them, 400 (4.5\%) cases received RP, while 8,463 (95.5\%) patients were treated with NLT. The median age at initial diagnosis was 69 years [interquartile range (IQR), 62-77 years]. The baseline clinicopathological characteristics of the entire patients are presented in Table 1. Patients with younger age had a lower PSA value, and harbored low-moderate disease grade tended to receive RP. Likewise, patients with larger tumors, more positive lymph nodes, and metastatic substage M1a were more likely to undergo RP.

Among the entire patients finally recruited, 4,285 (48.3\%) patients died of PCa during the follow-up (median 
Table 2 Multivariable competing risks regression analysis for cancer-specific mortality in patients received nonlocal treatment

\begin{tabular}{|c|c|c|}
\hline \multirow{2}{*}{ Variable } & \multicolumn{2}{|c|}{ Multivariate } \\
\hline & $\mathrm{HR}(95 \% \mathrm{Cl})$ & $P$ value \\
\hline \multicolumn{3}{|l|}{ Age, years } \\
\hline$<70$ & Ref. & \\
\hline$\geq 70$ & $1.01(0.95-1.07)$ & 0.760 \\
\hline LogPSA (ng/mL) & $1.41(1.30-1.53)$ & $<0.001$ \\
\hline \multicolumn{3}{|l|}{ Gleason score } \\
\hline$\leq 7$ & Ref. & \\
\hline$\geq 8$ & $1.58(1.46-1.70)$ & $<0.001$ \\
\hline \multicolumn{3}{|l|}{ T stage } \\
\hline $\mathrm{T} 1-3$ & Ref. & \\
\hline $\mathrm{T} 4$ & $1.31(1.20-1.44)$ & $<0.001$ \\
\hline \multicolumn{3}{|l|}{$\mathrm{N}$ stage } \\
\hline No & Ref. & \\
\hline $\mathrm{N} 1$ & $1.15(1.07-1.23)$ & $<0.001$ \\
\hline \multicolumn{3}{|l|}{ M stage } \\
\hline M1a & Ref. & \\
\hline M1b & $1.54(1.34-1.76)$ & $<0.001$ \\
\hline M1c & $1.78(1.54-2.06)$ & $<0.001$ \\
\hline
\end{tabular}

$\mathrm{HR}$, hazard ratio; $\mathrm{Cl}$, confidence interval; PSA, prostate-specific antigen. time: 28 months; IQR, 16-50 months). The 1-, 3-, and 5 -year cumulative incidence of CSM for the entire patients were $10.0 \%, 38.4 \%$, and $52.4 \%$, respectively. In the NLT patients, the multivariate competing risks analysis identified PSA value $(\mathrm{P}<0.001)$, Gleason score $(\mathrm{P}<0.001)$, $\mathrm{T}$ stage $(\mathrm{P}<0.001)$, pelvic lymphadenopathy $(\mathrm{P}<0.001)$, and metastatic substages $(\mathrm{M} 1 \mathrm{a}, \mathrm{M} 1 \mathrm{~b}$, or $\mathrm{M} 1 \mathrm{c})(\mathrm{P}<0.001)$ as independent predictors for CSM (Table 2). However, age had no significant effect on the survival outcome in patients with metastatic PCa. All predictors in the multivariable competing risks analyses model were then incorporated into prognostic nomogram model for CSM (Figure 1). Harrell's C index of the novel nomogram was 0.630 (95\% CI, 0.628-0.632), reflecting the moderate discriminatory power of the model. The AUC for the 1-, 3- and 5-year CSM was $0.624,0.616$ and 0.641 , respectively, reflecting a relatively favorable agreement in the probability of cancer-specific survival between the nomogram prediction and actual observation (Figure S1). Each variable in the predictive model was assigned a score according to its contributions as presented in the nomogram (Figure 1). Furthermore, according to the sum of points, we were easily able to determine the predicted probability of CSM for a patient at each time point.

Furthermore, based on the total scores of each patient in the NLT group produced by the nomogram, two cutoff values (score 125 and 183) were determined by using decision tree analysis (Figure 2). Then all patients were

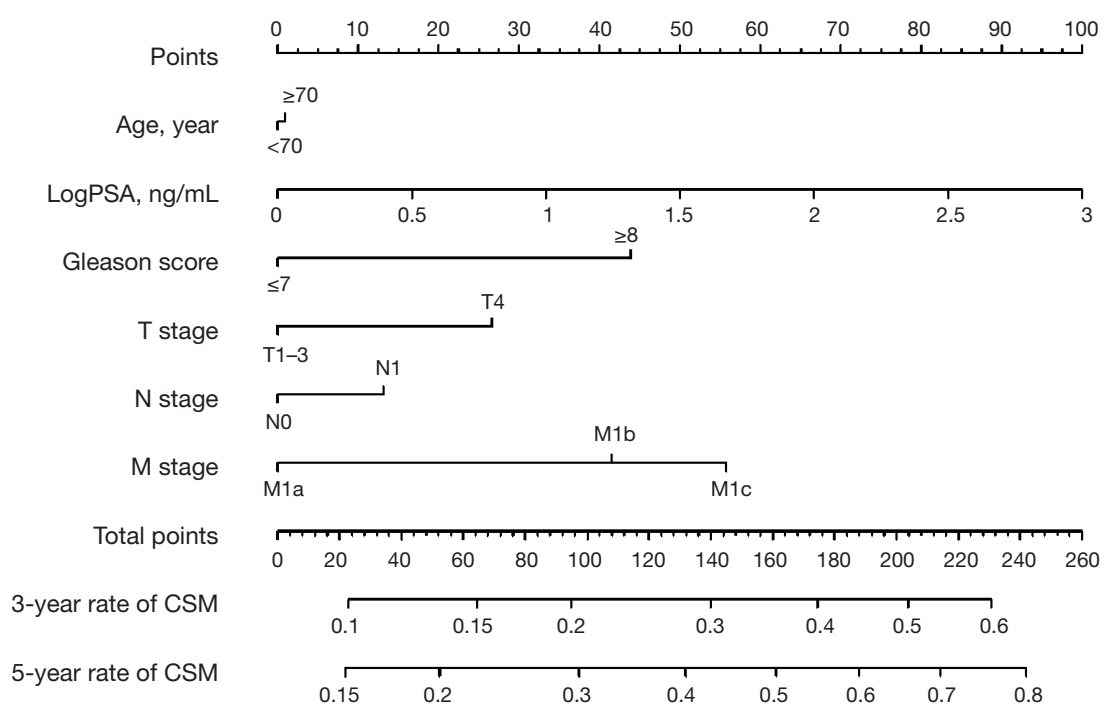

Figure 1 Nomogram predicting the CSM for metastatic prostate cancer patients received nonlocal treatment. PSA, prostate-specific antigen; CSM, cancer-specific mortality. 


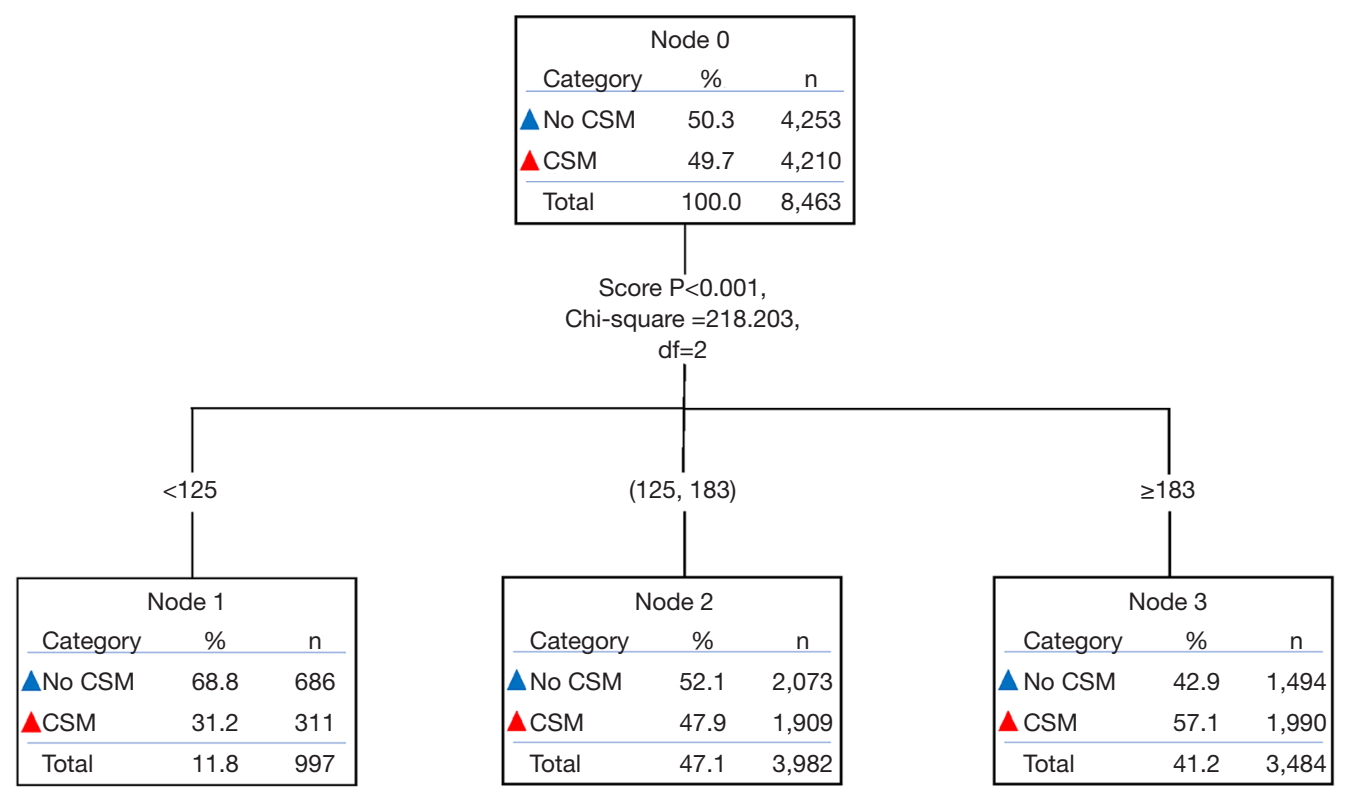

Figure 2 Decision tree analysis according to the effect of nomogram score on cancer-specific mortality in patients received nonlocal treatment. CSM, cancer-specific mortality.

Table 3 Effect of radical prostatectomy on cancer-specific mortality according to multivariate analysis in different groups

\begin{tabular}{lcccc}
\hline Group & Treatment type & 5-year cumulative incidence of CSM, \% & P value & HR (95\% Cl) \\
\hline All patients $(n=8,863)$ & RP & 20.4 & $<0.001$ & $0.396(0.315-0.498)$ \\
Low-risk $(n=1,171)$ & NLT & 53.8 & $<0.001$ & $0.231(0.134-0.397)$ \\
RP & NLT & 6.9 & $<0.001$ & $0.368(0.270-0.503)$ \\
High risk $(n=3,530)$ & RP & 28.6 & 27.1 & \\
& NLT & 52.1 & 0.190 & $0.748(0.485-1.150)$ \\
\hline
\end{tabular}

HR in multivariate analysis were adjusted for age, prostate-specific antigen (PSA), Gleason score, and tumor stage. CSM, cancer-specific mortality; HR, hazard ratio; $\mathrm{Cl}$, confidence interval; RP, radical prostatectomy; NLT, nonlocal treatment.

classified into three prognostic groups: patients with a total score $<125$ were sorted into the low-risk group ( $\mathrm{n}=1,171)$, cases with a total score $\geq 183$ were sorted into the high-risk group $(n=3,530)$, and the remaining patients were sorted into the intermediate-risk group $(n=4,162)$. In the total cohort, these three risk subgroups had a distinct difference in the 5-year cumulative incidence of CSM: $25.6 \%$ in the low-risk group, $51.1 \%$ in the intermediate-risk group, and $63.6 \%$ in the high-risk group, respectively $(\mathrm{P}<0.001$; Figure $\mathrm{S} 2)$.

For the total patients, RP was significantly associated with decreased CSM compared with NLT (5-year cumulative incidence $20.4 \%$ vs. $53.8 \%$; HR $=0.396,95 \%$ CI, 0.315-0.498; $\mathrm{P}<0.001$; Table 3; Figure 3). In subgroup analysis (Table 3; Figure 3), RP also demonstrated a significantly lower CSM in the low-risk group (5-year cumulative incidence $6.9 \%$ vs. $28.6 \%$; HR $=0.231,95 \%$ CI, $0.134-0.397 ; \mathrm{P}<0.001)$, and intermediate-risk group (5-year cumulative incidence $27.1 \%$ vs. $52.1 \%$; HR $=0.368,95 \%$ CI, 0.270-0.503; $\mathrm{P}<0.001)$. However, RP was not correlated with CSM in the high-risk group (5-year cumulative 

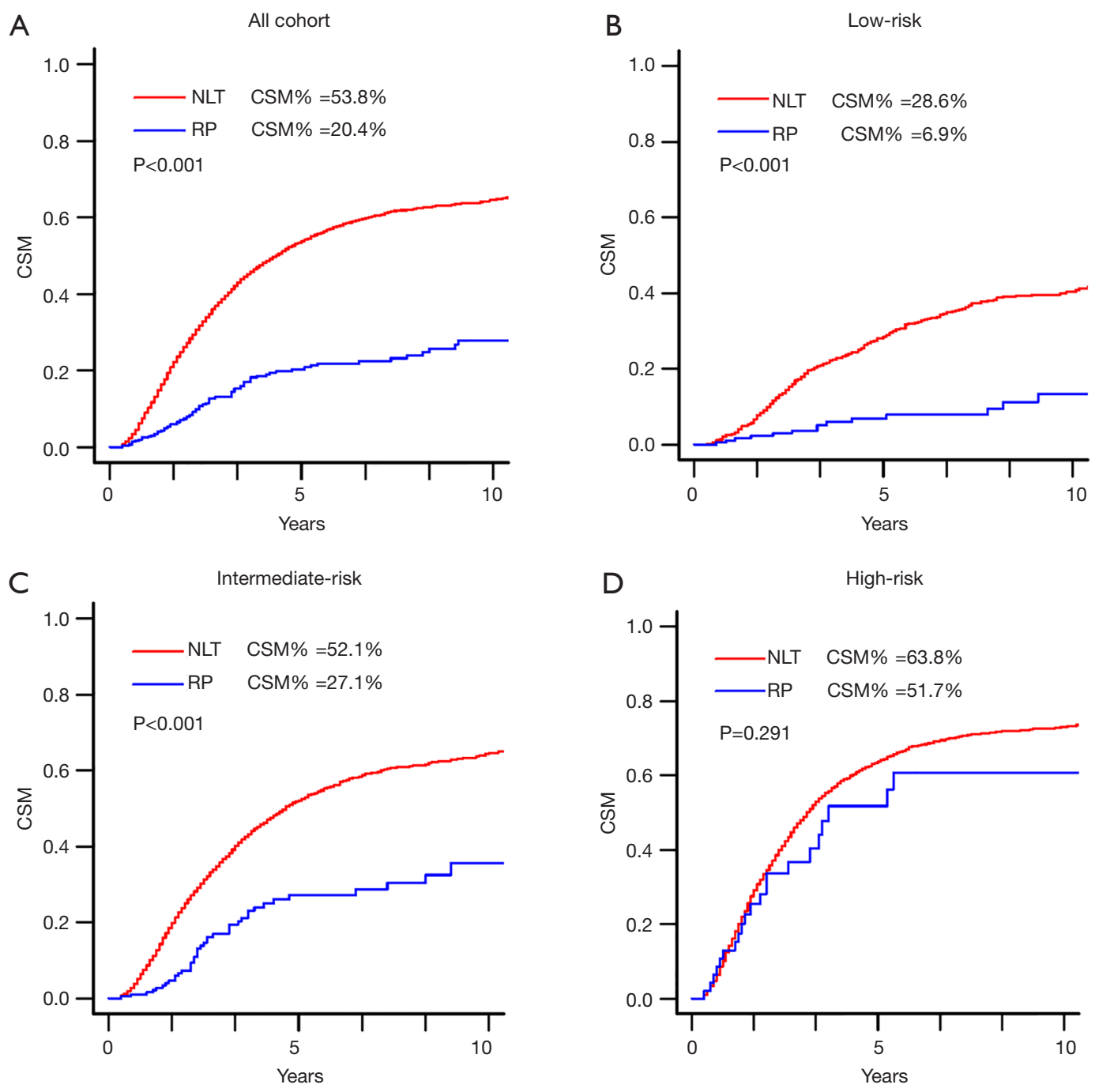

Figure 3 Cumulative incidence of CSM by RP in the entire cohort (A), low-risk group (B), intermediate-risk group (C) and high-risk group (D). CSM, cancer-specific mortality; RP, radical prostatectomy; NLT, nonlocal treatment.

incidence $51.7 \%$ vs. $63.8 \%$; HR $=0.748,95 \%$ CI, $0.485-$ $1.240 ; \mathrm{P}=1.190)$.

\section{Discussion}

Despite several large retrospective studies, the role of RP in patients with metastatic PCa remains controversial. Based on a population-based cohort, we established a novel competing risk nomogram and risk classification system to predict individuals' risk of CSM and identify the optimal candidates for RP in metastatic PCa patients. Patients in the low-risk group, and intermediate-risk group were found to benefit from RP, whereas RP did not confer any survival advantage in the high-risk group compared with NLT.

Advances in contemporary systemic therapy, specifically the introduction of novel agents, have shown improvement in survival for patients diagnosed with metastatic PCa (17-19). However, their survival remains invariably dismal, in stark contrast with men diagnosed without $\mathrm{mPCa}$, and the economic burden of these patients is significantly heavy (3). Conversely, the survival benefit from RP has shown very encouraging results. Previously mentioned several studies have explored and demonstrated that definitive local treatment (either RP, or radiation therapy) resulted in a significant lower mortality relative to NLT in patients with newly diagnosed metastatic PCa, regardless of CSM 
or overall mortality $(\mathrm{OM})(7-10)$. In the present study, we found that the 5-year cumulative incidence of CSM was $20.4 \%$ in the RP group and $53.8 \%$ in the NLT group, which further confirmed that patients with mPCa can benefit from RP.

Recently, several studies suggested that patient subsets with favorable oncological profiles at lower risk of CSM might benefit more from removal of the primary tumor. In a study based on the SEER database (20), RP was found to be invariably correlated with reduced CSM in patients with Gleason score $\leq 7, \leq \mathrm{cT} 3$, and M1a substage, reflecting patients with the lowest CSM rates were more likely to benefit from RP. In the present study, we noticed that RP was significantly associated with a $21.7 \%$ risk reduction in 5 -year cumulative incidence of CSM for patients in the lowrisk group and a $25.0 \%$ risk reduction in the intermediaterisk group. However, in the high-risk group, patients had a similar CSM rate regardless of whether they received RP or NLT. Similarly, a population-based study reported that only patients with a 3 -year predicted CSM risk $\leq 40 \%$, might benefit from LT, regardless of $\mathrm{RP}$, or radiation therapy (21). Likewise, a large hospital-based national cohort revealed that local treatment (either RP, or radiation therapy) could confer a survival benefit only when 3-year predicted OM risk was $<70 \%$ (9). These findings emphasize that the potential benefits of RP depend on patient's health condition and tumor characteristics, in addition, it is important to avoid over- or undertreatment by defining patient selection criteria in the metastatic scenario.

In general, most previous studies on comparing RP $v s$. NLT for metastatic PCa have certain limitations. First and foremost, most of these reports were limited by the small size of RP cohorts, implying patient selection bias. Moreover, due to the lack of information about EBRT in the SEER database, local therapy in several studies focused on brachytherapy. No studies on radiation and EBRT have been conducted, specifically without understanding the appropriate dose, to ensure that this is not palliative radiation. In addition, there are few studies that directly compare RP with NLT. This significantly limits our ability to understand this issue. Furthermore, most previous reports had some methodological limitations, such as confounding all-cause mortality rates, and lacking of competing risk regression analysis, which limits their validity. Because, in the presence of competing risks, the competing risk analysis has proven to be a reliable method and has been widely used in cancer research, compared with the standard Cox proportional hazards regression model
$(22,23)$.

In this study, based on the available predictors (age at diagnosis, PSA value, Gleason score, T stage, $\mathrm{N}$ stage, and metastatic substages), we developed a novel competing risk nomogram and a risk classification system to quantify the individuals' risk of CSM in patients with metastatic PCa. All predictors included in the predictive model can be easily obtained from clinical practices. Fossati et al. (21) also created a predictive model based on 7,569 metastatic $\mathrm{PCa}$ patients with NLT treatment identified from the SEER database (2004-2011). Although they included all predictors into the final model, the $\mathrm{C}$ index was only 0.61 , the followup time was relatively short, and without using competing risks analysis, which may limit the performance of their model. Unfortunately, despite all available predictive factors were incorporated into our model, the $\mathrm{C}$ index of the present nomogram for predicting CSM was 0.630, with moderate discriminatory power. This might be due to the lack of data regarding some important prognostic factors, such as systemic therapies, the extent metastasis, and salvage treatment, which have a great influence on disease progress and survival (24-26). The limitations of their model and current predictive model reveal the complexity of this issue, which needs to be clarified in ongoing clinical trials.

After risk prediction by the prognostic nomogram for CSM, risk classification is another pivotal step to identify optimal candidates for RP in patients with metastatic PCa. Relying on decision tree analysis, we identified two optimal cutoff values, then divided all patients into three different risk groups with a distinct CSM rate. Therefore, our approaches included predictive model and decision tree analysis and demonstrated its great performance in patient stratification, enabling clinicians to conveniently estimate individuals' risk of CSM and select suitable patients who are more likely to benefit from RP. This approach might be a meaningful and practical tool for addressing the issue about the role of RP in metastatic PCa.

There are several limitations in this study. First, despite this study is based on the SEER database and covers approximately $28 \%$ of the American population, the sample size of the RP group is relatively small and also limited by the inherent bias of a retrospective study. Second, information on patient performance status, comorbidities, complications of RP, which may be used for treatment decision-making. Third, detailed information on testosterone level, systemic treatment, radiation therapy, and the extents of distant metastasis are unavailable, which will undoubtedly influence the survival of metastatic PCa 
patients $(25,27)$. Fourth, in this cohort, we excluded patients who received radiation therapy, including EBRT and brachytherapy, because information regarding EBRT organ site-specific codes are not recorded, as well as we cannot ensure that this is not palliative radiation, failing to examine the impact of prostate-directed radiation therapy on patient survival. Lastly, the power of this prognostic nomogram is restricted by the lacking of sufficient information regarding some important risk factors, but the present predictive model may be used to improve the understanding of the benefits of RP in this setting, and is conveniently performed in clinical practices.

\section{Conclusions}

Utilizing prognostic nomogram and decision tree analysis, we developed a new model for individuals' risk prediction and corresponding risk classification in this setting. We identified patient subgroup at low or moderate risk of CSM may benefit more from RP. Conversely, RP did not confer any survival benefit in high-risk patients. Given the limited efficacy of contemporary systematic therapy, the value of $\mathrm{RP}$ in patients with metastatic PCa calls for revaluation, especially in ongoing clinical trial. As a novel model for selecting suitable candidates for RP among patients with metastatic $\mathrm{PCa}$, there is a definite need for further research to validate and improve the merits of our nomogram.

\section{Acknowledgments}

The authors appreciate the Surveillance, Epidemiology, and End Results database in providing high quality clinical data for our research.

Funding: This work was supported by the National Natural Science Foundation of China (Reference Number: 81672552), the Science and Technology Foundation of Sichuan Province (2017JY0226) and the 1.3.5 project for disciplines of excellence, West China Hospital, Sichuan University.

\section{Footnote}

Reporting Checklist: The authors have completed the TRIPOD reporting checklist. Available at http://dx.doi. org/10.21037/tau-20-1166

Peer Review File: Available at http://dx.doi.org/10.21037/ tau-20-1166
Conflicts of Interest: All authors have completed the ICMJE uniform disclosure form (available at http://dx.doi. org/10.21037/tau-20-1166). The authors have no conflicts of interest to declare.

Ethical Statement: The authors are accountable for all aspects of the work in ensuring that questions related to the accuracy or integrity of any part of the work are appropriately investigated and resolved. The study was conducted in accordance with the Declaration of Helsinki (as revised in 2013).

Open Access Statement: This is an Open Access article distributed in accordance with the Creative Commons Attribution-NonCommercial-NoDerivs 4.0 International License (CC BY-NC-ND 4.0), which permits the noncommercial replication and distribution of the article with the strict proviso that no changes or edits are made and the original work is properly cited (including links to both the formal publication through the relevant DOI and the license). See: https://creativecommons.org/licenses/by-nc-nd/4.0/.

\section{References}

1. Weiner AB, Matulewicz RS, Eggener SE, et al. Increasing incidence of metastatic prostate cancer in the United States (2004-2013). Prostate Cancer Prostatic Dis 2016;19:395-7.

2. Cornford P, Bellmunt J, Bolla M, et al. EAU-ESTROSIOG Guidelines on Prostate Cancer. Part II: Treatment of Relapsing, Metastatic, and Castration-Resistant Prostate Cancer. Eur Urol 2017;71:630-42.

3. Dragomir A, Dinea D, Vanhuyse M, et al. Drug costs in the management of metastatic castration-resistant prostate cancer in Canada. BMC Health Serv Res 2014;14:252.

4. Bristow RE, Tomacruz RS, Armstrong DK, et al. Survival effect of maximal cytoreductive surgery for advanced ovarian carcinoma during the platinum era: a metaanalysis. J Clin Oncol 2002;20:1248-59.

5. Esquivel J, Sticca R, Sugarbaker P, et al. Cytoreductive surgery and hyperthermic intraperitoneal chemotherapy in the management of peritoneal surface malignancies of colonic origin: a consensus statement. Society of Surgical Oncology. Ann Surg Oncol 2007;14:128-33.

6. Flanigan RC, Salmon SE, Blumenstein BA, et al. Nephrectomy followed by interferon alfa-2b compared with interferon alfa-2b alone for metastatic renal-cell cancer. N Engl J Med 2001;345:1655-9.

7. Culp SH, Schellhammer PF, Williams MB. Might Men 
Diagnosed with Metastatic Prostate Cancer Benefit from Definitive Treatment of the Primary Tumor? A SEERBased Study. Eur Urol 2014;65:1058-66.

8. Satkunasivam R, Kim AE, Desai M, et al. Radical Prostatectomy or External Beam Radiation Therapy vs No Local Therapy for Survival Benefit in Metastatic Prostate Cancer: A SEER-Medicare Analysis. J Urol 2015;194:378-85.

9. Loppenberg B, Dalela D, Karabon P, et al. The Impact of Local Treatment on Overall Survival in Patients with Metastatic Prostate Cancer on Diagnosis: A National Cancer Data Base Analysis. Eur Urol 2017;72:14-9.

10. Gratzke C, Engel J, Stief CG. Role of Radical Prostatectomy in Metastatic Prostate Cancer: Data from the Munich Cancer Registry. Eur Urol 2014;66:602-3.

11. Sooriakumaran P, Karnes J, Stief C, et al. A Multiinstitutional Analysis of Perioperative Outcomes in 106 Men Who Underwent Radical Prostatectomy for Distant Metastatic Prostate Cancer at Presentation. Eur Urol 2016;69:788-94.

12. Heidenreich A, Pfister D, Porres D. Cytoreductive Radical Prostatectomy in Patients with Prostate Cancer and Low Volume Skeletal Metastases: Results of a Feasibility and Case-Control Study. J Urol 2015;193:832-8.

13. Yuh BE, Kwon YS, Shinder BM, et al. Results of Phase 1 study on cytoreductive radical prostatectomy in men with newly diagnosed metastatic prostate cancer. Prostate Int 2019;7:102-7.

14. Luo C, Zhong X, Deng L, et al. Nomogram Predicting Locoregional Recurrence to Assist Decision-Making of Postmastectomy Radiation Therapy in Patients With T1-2N1 Breast Cancer. Int J Radiat Oncol Biol Phys 2019;103:905-12.

15. Zaak D, Burger M, Otto W, et al. Predicting individual outcomes after radical cystectomy: an external validation of current nomograms. BJU Int 2010;106:342-8.

16. Pinart M, Kunath F, Lieb V, et al. Prognostic models for predicting overall survival in metastatic castrationresistant prostate cancer: a systematic review. World J Urol 2020;38:613-35.

Cite this article as: $\mathrm{Wu} \mathrm{K}$, Tang Y, Shao Y, Li X. Nomogram predicting survival to assist decision-making of radical prostatectomy in patients with metastatic prostate cancer. Transl Androl Urol 2021;10(2):879-887. doi: 10.21037/tau-20-1166
17. de Bono JS, Logothetis CJ, Molina A, et al. Abiraterone and increased survival in metastatic prostate cancer. $\mathrm{N}$ Engl J Med 2011;364:1995-2005.

18. de Bono JS, Oudard S, Ozguroglu M, et al. Prednisone plus cabazitaxel or mitoxantrone for metastatic castrationresistant prostate cancer progressing after docetaxel treatment: a randomised open-label trial. Lancet 2010;376:1147-54.

19. Scher HI, Fizazi K, Saad F, et al. Increased survival with enzalutamide in prostate cancer after chemotherapy. $\mathrm{N}$ Engl J Med 2012;367:1187-97.

20. Leyh-Bannurah SR, Gazdovich S, Budaus L, et al. Local Therapy Improves Survival in Metastatic Prostate Cancer. Eur Urol 2017;72:118-24.

21. Fossati N, Trinh QD, Sammon J, et al. Identifying optimal candidates for local treatment of the primary tumor among patients diagnosed with metastatic prostate cancer: a SEER-based study. Eur Urol 2015;67:3-6.

22. Carmona R, Zakeri K, Green G, et al. Improved Method to Stratify Elderly Patients With Cancer at Risk for Competing Events. J Clin Oncol 2016;34:1270-7.

23. Zhang Z, Geskus RB, Kattan MW, et al. Nomogram for survival analysis in the presence of competing risks. Ann Transl Med 2017;5:403.

24. Ost P, Decaestecker K, Lambert B, et al. Prognostic factors influencing prostate cancer-specific survival in noncastrate patients with metastatic prostate cancer. Prostate 2014;74:297-305.

25. Halabi S, Kelly WK, Ma H, et al. Meta-Analysis Evaluating the Impact of Site of Metastasis on Overall Survival in Men With Castration-Resistant Prostate Cancer. J Clin Oncol 2016;34:1652-9.

26. Gandaglia G, Karakiewicz PI, Briganti A, et al. Impact of the Site of Metastases on Survival in Patients with Metastatic Prostate Cancer. Eur Urol 2015;68:325-34.

27. James ND, Sydes MR, Mason MD, et al. Celecoxib plus hormone therapy versus hormone therapy alone for hormone-sensitive prostate cancer: first results from the STAMPEDE multiarm, multistage, randomised controlled trial. Lancet Oncol 2012;13:549-58. 


\section{Supplementary}
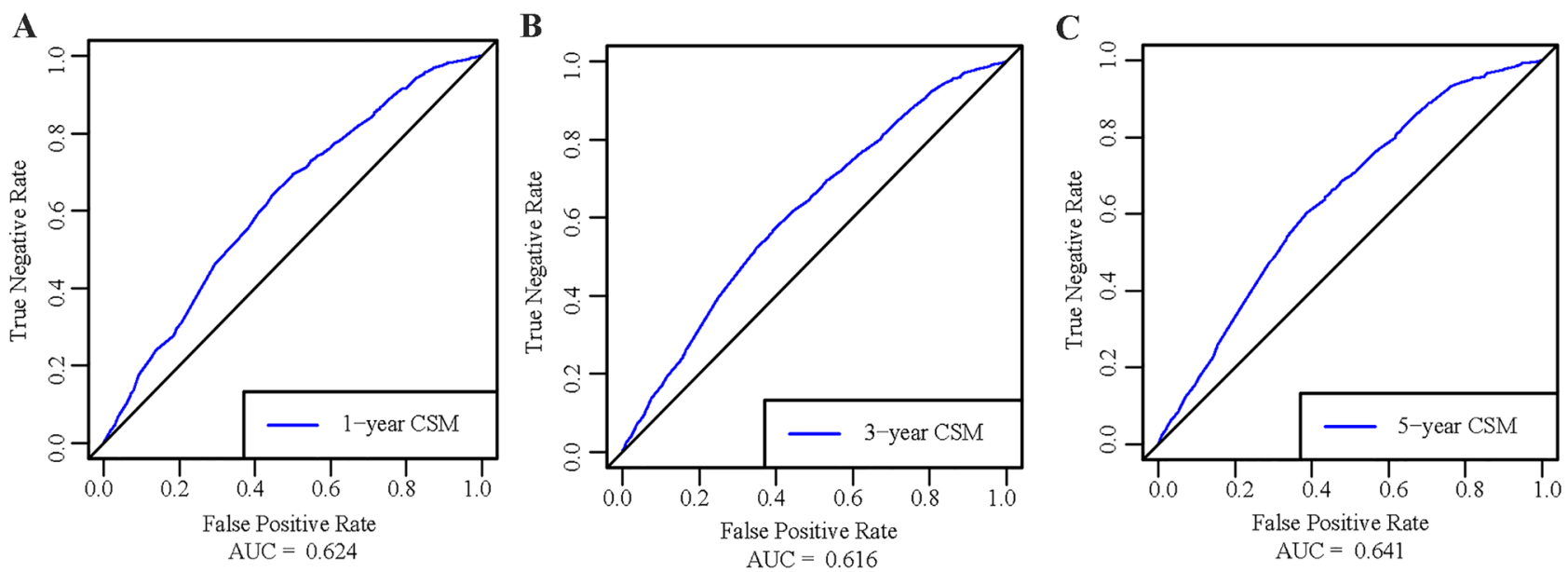

Figure S1 Area under the curves to predict CSM at 1 year (A), 3 years (B) and 5 years (C). CSM, cancer-specific mortality.

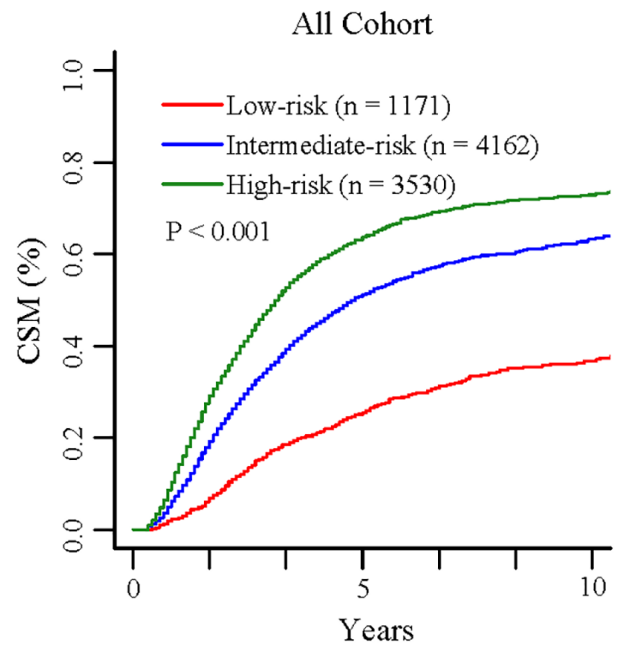

Figure S2 Cumulative incidence of CSM for the entire patients in the low-, intermediate-, and high-risk groups. CSM, cancer-specific mortality. 